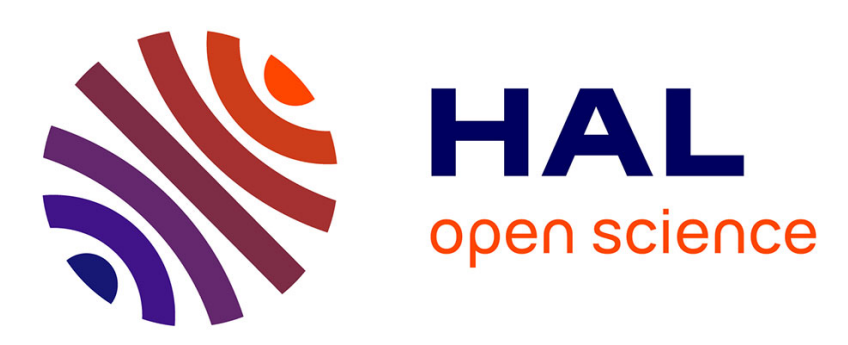

\title{
Tomographic Diffractive Microscopy: Basics, techniques and perspectives
}

Olivier Haeberlé, Kamal Belkebir, H. Giovaninni, Anne Sentenac

\section{To cite this version:}

Olivier Haeberlé, Kamal Belkebir, H. Giovaninni, Anne Sentenac. Tomographic Diffractive Microscopy: Basics, techniques and perspectives. Journal of Modern Optics, 2010, 57 (9), pp.686-699. 10.1080/09500340.2010.493622 . hal-00827966

\section{HAL Id: hal-00827966 https://hal.science/hal-00827966}

Submitted on 30 May 2013

HAL is a multi-disciplinary open access archive for the deposit and dissemination of scientific research documents, whether they are published or not. The documents may come from teaching and research institutions in France or abroad, or from public or private research centers.
L'archive ouverte pluridisciplinaire HAL, est destinée au dépôt et à la diffusion de documents scientifiques de niveau recherche, publiés ou non, émanant des établissements d'enseignement et de recherche français ou étrangers, des laboratoires publics ou privés. 


\title{
Tomographic Diffractive Microscopy: Basics, techniques and perspectives
}

\author{
O. Haeberlé ${ }^{1}$ K. Belkebir ${ }^{2}$, H. Giovaninni ${ }^{2}$, A. Sentenac ${ }^{2}$ \\ ${ }^{1}$ Laboratoire MIPS-EA2332, Université de Haute-Alsace, IUT Mulhouse, 61 rue \\ Albert Camus, 68093 Mulhouse Cedex, France \\ ${ }^{2}$ Institut Fresnel, Aix-Marseille Université, CNRS, Campus de St Jérôme, 13013 \\ Marseille France
}

corresponding author: olivier.haeberle@uha.fr or anne.sentenac@fresnel.fr

(Received XXXX; final version received $X X X X X)$

\begin{abstract}
Tomographic Diffractive Microscopy (TDM) is an advanced digital imaging technique, which combines the recording of multiple holograms with the use of inversion procedures to retrieve quantitative information on the sample. In this review, we discuss the basic theory of TDM in the framework of electromagnetism and draw a comparison with conventional widefield microscopes. We describe various implementations of TDM, highlighting their power of resolution. Finally, we present some research perspectives for increasing the potential of this promising new imaging modality.
\end{abstract}

Keywords: microscopy, diffraction, holograms, imaging, resolution, inversion, tomography

\section{Introduction}

The optical microscope has become an invaluable tool for biology thanks to its unique capabilities to image living specimens in three dimensions, and over long periods (time-lapse microscopy), due to the non-ionizing nature of light. Fluorescence techniques are particularly appreciated because they allow for specific labelling of cellular structures. However, fluorescent markers may induce unfavourable effects like photo-toxicity and they do not permit an overall imaging of the sample.

As a consequence, among the many techniques which have been developed, those permitting to observe a specimen without the need for specific staining have known a regain of interest in recent years. One may cite, for example, SecondHarmonic Generation microscopy (SHG), Coherent Anti-Stokes Raman Spectroscopy microscopy (CARS) and conventional transmission microscopy. In transmission microscopy (classical, phase-contrast or Differential Interference Contrast (DIC)), the image is formed by a complex interaction of the incoherent illuminating light with the 
specimen. The recorded contrast, while very helpful for morphological studies, does not yield quantitative information on the opto-geometrical characteristics of the sample. In particular, the optical index of refraction distribution within the specimen is difficult to reconstruct.

On the contrary, the use of coherent light illumination, combined with interferometric detection, permits to record holograms, which encode both the amplitude and phase of the light diffracted by the specimen. Using an adapted model of diffraction (typically the first order Born approximation), this so-called holographic microscopy allows for the reconstruction of the specimen index of refraction distribution. When this technique is combined with a rotation of the specimen or an inclination of the illumination wave, the set of recorded holograms represents a diffractive tomographic acquisition, which permits 3-D reconstructions of much better quality.

In the last five years, there have been a growing number of papers on novel microscopy techniques that combine numerical reconstruction processes and sophisticated holographic experimental set-ups to retrieve with a high resolution the opto-geometrical parameters of non-fluorescent samples. These approaches are known under various names, synthetic aperture microscopy, tomographic phase microscopy, optical diffraction tomography, digital holographic microscopy, scanning holography microscopy etc. They are dedicated to various applications (3D reconstruction of the refraction index of biological samples, profilometry of manufactured nanocomponents, rugosimetry, etc). They usually present a higher precision and/or resolution standard optical microscopes and/or they allow for the retrieval of quantitative information on the sample. This review aims at gathering all these emerging digital imaging techniques in a common framework, which we call 
Tomographic Diffractive Microscopy (TDM). We first briefly recall the theoretical basis of optical digital imaging, then give an overview of the different techniques that pertains to TDM, and finally present some research perspectives.

\section{Basics of diffraction and application to imaging}

Digital imaging techniques rely on inversion algorithms to retrieve the parameters of interest of the sample. Hence, they require an accurate modelling of the link between the measured data and the sample. In this section, we first recall the basics in waveobject interaction and give the general principles of Tomographic Diffractive Microscopy (TDM). We present a short analysis of its resolution, compared to that of wide-field optical microscopes.

\subsection{Basics of diffraction}

The aim of this paragraph is to recall the relationship between the opto-geometrical characteristics of an object and its diffracted field. This link is at the core of most imaging systems that use waves for probing samples. For the sake of simplicity, we restrict the discussion to the scalar approximation. Accounting for the vector nature of electromagnetic waves does not change the main lines of the presentation. We refer the interested reader willing to complete this rapid introduction to textbooks on electromagnetism or wave diffraction, as for example [1].

We consider in Fig. 1, an object in vacuum defined by its relative permittivity $\varepsilon(\mathbf{r})$ and illuminated by a monochromatic incident wave, with wavelength $\lambda=2 \pi c / \omega$ stemming from a source $S(\mathbf{r})$. Hereafter, the $\exp (-i \omega t)$ dependence is omitted. The total scalar field satisfies the Helmholtz equation:

$$
\Delta E(\mathbf{r})+k_{0}^{2} E(\mathbf{r})=X(\mathbf{r}) k_{0}^{2} E(\mathbf{r})+S(\mathbf{r})
$$


where $k_{0}$ is the wavenumber $2 \pi / \lambda$, and the contrast of permittivity $X=1$ -

$\varepsilon$. Introducing the Green function,

$$
G(\mathbf{r})=-\exp \left(i k_{0} r\right) / 4 \pi r
$$

we obtain the integral equation for the total field,

$$
E(\mathbf{r})=E_{\text {inc }}(\mathbf{r})+k_{0}^{2} \int G\left(\mathbf{r}-\mathbf{r}^{\prime}\right) X\left(\mathbf{r}^{\prime}\right) E\left(\mathbf{r}^{\prime}\right) d \mathbf{r}^{\prime}
$$

where $E_{\text {inc }}$ is the field generated by $S$ that would exist in the absence of the

object. The support of the integral in (3) is limited to the geometrical support $\Omega$ of the object. When the observation point $\mathbf{r}$ is far from the object, $r^{\prime 2} / \lambda<<r$, with $\mathbf{r}^{\prime}$ in $\Omega$, the diffracted field can be written as,

$$
E_{d}(\mathbf{r})=-\frac{\exp \left(i k_{0} r\right)}{4 \pi r} e(\mathbf{k})
$$

where the scattered far-field amplitude reads,

$$
e(\mathbf{k})=k_{0}^{2} \int \exp \left(-i \mathbf{k} \cdot \mathbf{r}^{\prime}\right) X\left(\mathbf{r}^{\prime}\right) E\left(\mathbf{r}^{\prime}\right) d \mathbf{r}^{\prime}
$$

We now suppose that the incident field is a plane wave with wavevector $\mathbf{k}_{\text {inc }}$, $E_{i n c}(\mathbf{r})=A_{\text {inc }} \exp \left(i \mathbf{k}_{\text {inc }} . \mathbf{r}\right)$, and that the object is weakly diffracting, so that the field inside $\Omega$ is close to $E_{\text {inc }}$. Thus, the Born approximation is assumed to be valid [2]. In this case, Eq. (5) reduces to, ${ }^{1}$

$$
e\left(\mathbf{k}, \mathbf{k}_{\text {inc }}\right)=C \tilde{X}\left(\mathbf{k}-\mathbf{k}_{\text {inc }}\right)
$$

where $C=8 \pi^{3} A_{\text {inc }} k_{0}^{2}$. Equation (6) provides a one to one correspondence between the diffracted far-field amplitude and the Fourier coefficient of the relative permittivity of the object. It is at the basis of most far-field imaging technique such as X-ray diffraction tomography [3], acoustic tomography [4] and, as will be seen later, Tomographic Diffractive Microscopy [5]. Note that an expression similar to Eq. (6) is obtained in the vectorial case by replacing $C$ by a vector, whose direction is given by the projection of the incident field polarization $\mathbf{A}_{\text {inc }}$ onto the plane normal to the wavevector $\mathbf{k}[1]$.

\footnotetext{
${ }^{1}$ Throughout this paper, the Fourier transform is defined by, $g(\mathbf{r})=\int \tilde{g}(\mathbf{i}) \exp (i \mathbf{i} . \mathbf{r}) d \mathbf{i} ; \tilde{g}(\mathbf{i})=\frac{1}{8 \pi^{3}} \int g(\mathbf{r}) \exp (-i \mathbf{i} . \mathbf{r}) d \mathbf{r}$
} 
It is possible to further simplify the relationship between the diffracted field and the optical properties of the object under the paraxial configuration by introducing the notion of "reflectance" or "transmittance". We consider an imaging system with a transverse plane $(x \mathrm{O} y)$ and an optical or axial axis $z$, Fig. 2.

One introduces $\mathbf{k}_{\|}$and $\mathbf{k}_{\text {inc } \|}$ the projections of the diffracted and incident wavevectors onto the transverse (xOy) plane and assumes that the vector components $k_{\mathrm{z}}$ and $k_{\text {inc, }}$ along the $z$ axis can be approximated by $k_{0}$. In other terms, the diffracted and incident directions are close to the optical axis of the imaging system, which amounts to the paraxial approximation. Applying the Born approximation to Eq. (5) and splitting the three-dimensional integral into a two-dimensional integral over the transverse plane and a one-dimensional integral over $z$, yields,

$$
e\left(\mathbf{k}, \mathbf{k}_{i n c}\right) \approx e\left(\mathbf{k}_{\|}, \mathbf{k}_{i n c \mid}\right)=\int \exp \left(-i \mathbf{k}_{\|}, \mathbf{r}_{\mid}\right) R\left(\mathbf{r}_{\mid}\right) \exp \left(i \mathbf{k}_{i n c \mid}, \mathbf{r}_{\|}\right) d \mathbf{r}_{\|}
$$

where the reflectance $R\left(\mathbf{r}_{\|}\right)$or transmittance $T\left(\mathbf{r}_{\|}\right)$represent the integral over $z$. Depending on the approximate models that are used for the wave-object interaction, different expressions for the reflectance or transmittance, associated to different domains of validity, are obtained. For example, if the sample is a homogeneous rough surface, the diffracted far-field can be written as a surface integral involving the surface currents at $\left[\mathbf{r}_{\|}, h\left(\mathbf{r}_{\|}\right)\right]$where $h$ is the height of the surface. Using Kirchhoff approximation [6] combined with paraxial configuration, one obtains an expression for $e\left(\mathbf{k}, \mathbf{k}_{\text {inc }}\right)$ similar to Eq. (7) where the reflectance is proportional to $\exp \left(2 \mathrm{i} k_{0} h\left(\mathbf{r}_{\|}\right)\right)$. Note however that, in all cases, the appearance of the 2-D Fourier transform over the transverse plane requires the paraxial approximation. Equation (7) is at the core of most synthetic aperture holographic imaging inverse techniques that are applied to rough surfaces or phase mask imaging $[7,8]$. 
From this short analysis of the diffraction process, one observes that if the Born approximation or the single scattering approximation is valid, the diffracted field is linearly related to the optogeometrical parameters of the object. On the contrary, if the sample supports multiple scattering, the inverse problem is non linear and necessitates to solve the volume integral equation given by Eq. (3). Most imaging setups in optics assume that the Born approximation or an equivalent single scattering approximation is valid.

\subsection{Principles of Tomographic Diffractive Microscopy and analysis of the resolution}

Basically, a Tomographic Diffractive Microscopy experiment consists in illuminating sequentially a sample by a plane wave with varying wavevector $\mathbf{k}_{\text {inc }}$ and measuring, for each illumination, the far-field diffracted along various directions of observation, denoted by the wavevector $\mathbf{k}$. The map of permittivity of the object is then retrieved from the set of complex data $e\left(\mathbf{k}, \mathbf{k}_{\text {inc }}\right)$. The one-to-one correspondence between $e\left(\mathbf{k}, \mathbf{k}_{\text {inc }}\right)$ and $\tilde{X}\left(\mathbf{k}-\mathbf{k}_{\text {inc }}\right)$, which exists under the Born approximation, permits to easily evaluate the resolution of such an imaging system. It is given by the threedimensional Fourier domain of the permittivity that is accessible with the incident and observation angular spans. In the best case, when the illumination and the observation are performed all around the sample, the Fourier coefficient of the permittivity can be measured within the entire sphere of radius $2 k_{0}$ depicted in Fig. 3. This sphere is known as the Ewald sphere. The permittivity that can be estimated with the imaging system is given by,

$$
X^{\text {estimation }}=X \otimes g
$$

where the point spread function $g$ indicates that the Fourier transform of $X$ is known in a bounded spectral domain only. The Point Spread Function $g$ is the Fourier Transform of the low-pass filter that is equal to one in the accessible Fourier domain 
and zero elsewhere. When the accessible Fourier domain is the sphere with radius $2 k_{0}$, one obtains,

$$
g(r)=\frac{4 \pi}{r^{3}}\left[\sin \left(2 k_{0} r\right)-2 k_{0} r \cos \left(2 k_{0} r\right)\right]
$$

The first zero of this function, which gives the resolution of the imaging set-up following the Rayleigh criterion, is obtained for $r \approx 0.35 \lambda$.

The previous analysis can be easily adapted to the retrieval of reflectance or transmittance, which are linked to the diffracted field via Eq. (7). One considers an imaging set-up where the transverse incident and observation wavenumbers, $k_{\|}$and $k_{\text {inc }}$, do not exceed $k_{0} \sin \theta$ where $\theta$ is small enough for the paraxial approximation to apply. In this case, the Fourier transform of the reflectance is known within a disk of radius $2 k_{0} \sin \theta$. The point spread function is then given by:

$$
g\left(r_{\|}\right)=2 \pi\left(2 k_{0} \sin \theta\right)^{2} J_{1}\left(2 k_{0} \sin \theta r_{\|}\right) /\left(2 k_{0} \sin \theta r_{\|}\right) .
$$

The Rayleigh transverse resolution is about $0.3 \lambda / N A$ where the numerical aperture $N A$ is defined by $\sin \theta$.

\subsection{Comparison between Tomographic Diffractive Microscopy and conventional wide-field microscopy}

In this paragraph, we present a short comparison between a Tomographic Diffractive

Microscopy set-up and a classical wide-field microscope with same numerical aperture. To simplify the analysis, we consider the issue of retrieving the twodimensional transmittance (instead of the three-dimensional permittivity) of a sample. A complete description of the three-dimensional imaging properties of a transmission microscope can be found in the paper by Streibl [9].

We consider the telecentric system described in Fig. 2. The sample is placed before the object focal plane of the objective and the field intensity is recorded on a camera placed at the image focal plane of the tube lens. In this mounting, the object focal plane of the tube lens merges with the image focal plane of the objective. In 
most wide-field microscopes, the illuminating source is a thermal lamp, which, in combination with a so-called condenser, generates a homogeneous illumination that can be seen as the sum of incoherent plane waves impinging on the sample with different directions. Due to the incoherence properties of the incident field, the detected intensity on the camera can be considered as the sum of the field intensities obtained for each incident plane wave. Hence, we will first consider the case where the object is illuminated by a unique plane wave with transverse wavevector $\mathbf{k}_{\text {inc }}$ and amplitude $A_{\text {inc }}$.

The formation of the image in a microscope relies on the analogical Fourier transform that is performed by the lenses. Indeed, under certain conditions, one can show that the field existing at the image focal plane of a lens is proportional to the Fourier transform of the field existing at the object focal plane. Hence, in the set-up presented in Fig. 4, one verifies that the field existing in the image plane of the tube lens, namely the CCD plane, will be equal to that existing at the object focal plane of the objective, i. e. very close to the sample, with a magnification equal to the ratio between the focal lengths of the tube lens and objective. Yet, the field recovery is incomplete due to the loss of information stemming from propagation and the finite collection cone of the objective. Hereafter, we call $\theta$ the collection angle of the objective and defines the numerical aperture of the microscope through $\sin \theta=N A$. For the sake of simplicity, we consider a microscope in air with a magnification equal to one. The imaging system acts as low-pass filter, symbolized by the pupil placed in the Fourier plane of the objective that cuts all the transverse Fourier components of the field that are above $k_{0} \sin \theta$. More precisely,

- at the object plane of the objective, the field $E_{O}$, can be written as a Fourier integral,

$$
E_{O}\left(\mathbf{r}_{\|}\right)=A_{\text {inc }} \exp \left(i \mathbf{k}_{\text {inc } \|}, \mathbf{r}_{\|}\right)+\int \tilde{E}\left(\mathbf{k}_{\|}, \mathbf{k}_{i n c \|}\right) \exp \left(i \mathbf{k}_{\|}, \mathbf{r}_{\|}\right) d \mathbf{k}_{\|}
$$


- at the Fourier plane, the field is proportional to $\tilde{E}\left(\mathbf{k}_{\|}, \mathbf{k}_{i n c \mid}\right)+A_{i n c} \delta\left(\mathbf{k}_{\|}-\mathbf{k}_{\text {inc } \|}\right)$ for $k_{\|}<k_{0} N A$ and 0 elsewhere;

- at the image plane, the field $E_{I}$ can be cast in the form:

$$
E_{I}\left(\mathbf{r}_{\|}\right)=p\left(\mathbf{k}_{i n c \mid}\right) A_{i n c} \exp \left(i \mathbf{k}_{i n c \|}, \mathbf{r}_{\|}\right)+\int p\left(\mathbf{k}_{\|}\right) \tilde{E}\left(\mathbf{k}_{\|}, \mathbf{k}_{i n c \|}\right) \exp \left(i \mathbf{k}_{\mid}, \mathbf{r}_{\|}\right) d \mathbf{k}_{\|}
$$

where $p(\mathbf{u})$ indicates the filtering function of the imaging set-up. $p(\mathbf{u})$ is a

radial function equal to zero if $u>k_{0} N A$ and one elsewhere.

From Eq. (12) and Eq. (4) in far-field, and using the stationary phase theorem, one shows that $\widetilde{E}\left(\mathbf{k}_{\|} \mathbf{k}_{\text {inc } \|}\right)=e\left(\mathbf{k}, \mathbf{k}_{\text {inc }}\right) /\left(i 8 \pi^{2} k_{z}\right)$. Hence, measuring the complex field in the Fourier plane of a microscope (or measuring the complex field in the image plane of the microscope and performing a numerical Fourier transform), permits to retrieve the far-field amplitude $e\left(\mathbf{k}, \mathbf{k}_{\text {inc }}\right)$ within the limited solid angle defined by the numerical aperture of the objective. This property is used in most tomographic diffractive microscope set-ups to retrieve the diffracted far-field for each illumination angle. On the other hand, in a conventional microscope, one detects the field intensity $\left|E_{I}\right|^{2}$, i. e. the interference between the incident and the diffracted fields. In this case, the relation-ship between the measured intensity and the optogeometrical characteristics of the objects is not simple as will be shown below.

Using Eq. (7), we first relate the Fourier transform of the field to the transmittance through, $\tilde{E}\left(\mathbf{k}_{\|}, \mathbf{k}_{i n c \mid}\right) \approx-i \widetilde{T}\left(\mathbf{k}_{\mid}-\mathbf{k}_{i n c \mid}\right) /(2 \pi)$. Neglecting the square of the diffracted field in $\left|E_{I}\right|^{2}$ yields,

$$
\left|E_{I}\left(\mathbf{r}_{\mid}\right)\right|^{2} \approx p\left(\mathbf{k}_{i n c \mid}\right)\left|A_{i n c}\right|^{2}-\frac{1}{2 \pi} \operatorname{Im} \int p\left(\mathbf{k}_{\|}\right) \widetilde{T}\left(\mathbf{k}_{\|}-\mathbf{k}_{\text {inc } \mid}\right) \exp \left[i\left(\mathbf{k}_{\|}-\mathbf{k}_{\text {inc } \|}\right) \mathbf{r}_{\|}\right] d \mathbf{k}_{\|}
$$

With an incoherent illumination, the detected intensity is the sum of $\left|E_{l}\right|^{2}$ for all possible $\mathbf{k}_{\text {inc } \|}$. We assume that the illumination angular span is the same as the detection angular span. Simple calculations yield,

$$
\left|E_{I}^{i n c o h}\left(\mathbf{r}_{\|}\right)\right|^{2} \approx A-4 \pi^{2} \operatorname{Im}(T \otimes g)\left(\mathbf{r}_{\mid}\right) \text {where } g\left(r_{\mid}\right)=\left[2 \pi\left(k_{0} \sin \theta\right)^{2} J_{1}\left(k_{0} \sin \theta r_{\|}\right) /\left(k_{0} \sin \theta r_{\|}\right)\right]^{2} .
$$


From this formula, one retrieves the classical Rayleigh criterion that states that two point-like objects will be distinguished within the image only if their interdistance is larger than $0.6 \lambda N A$. Note that the image is related to the imaginary part of the transmittance only. The relationship between the image and the object is even more complicated if one tries to connect the three-dimensional images (obtained by moving the CCD camera along the optical axis) to the three-dimensional permittivity of the sample [9]. Indeed, it appears that the point spread function is different for purely absorbing objects and purely dephasing objects. Hence, it is not possible to retrieve quantitatively the permittivity of the sample from the intensity measurements.

Comparing the point-spread functions obtained with the Tomographic Diffractive Microscopy set-up, Eq. (11) to that of the wide-field microscope with same illumination and detection angular spans, Eq. (15), one observes that the TDM resolution is twice better than that of the conventional microscope. However, it is important to stress that the Fourier support of both point-spread functions is the same, being a disk with radius $2 k_{0} N A$. The difference comes from the fact that the optical transfer function of the wide-field microscope tend towards 0 when $v$ tends toward $2 k_{0} N A$ while that of the TDM set-up is constant throughout this disk.

Note that this simple analysis holds true only if one assumes an infinite signal to noise ratio. In practice, the object spatial frequencies are not transmitted equally by the TDM imager towards the CCD plane. Indeed, one notes that a peculiar spatial frequency of the object contrast, $\tilde{X}(\mathbf{v})$, can be obtained with many pairs of diffracted and incident directions, $\left(\mathbf{k}, \mathbf{k}_{\text {inc }}\right)$. It is thus possible to reduce the noise by averaging the redundant measurements. Now, the smaller the spatial frequency $v$, the larger the number of pairs $\left(\mathbf{k}, \mathbf{k}_{\text {inc }}\right)$ such that $\left|\mathbf{k}-\mathbf{k}_{\text {inc }}\right|=v$. With this trivial data processing, the 
noise corrupting the low spatial frequencies is much lower than that corrupting the high spatial frequencies.

The comparison between a wide-field microscope and a Tomographic Diffractive Microscopy experiment with the same illumination and observation angular spans permits to stress the pros and cons of each technique. In a wide-field microscope, the sample is illuminated simultaneously under all the possible incident angles and $a$ single intensity measurement with a CCD camera provides an analogical image of the object in real time. Yet, this image cannot be linked in a simple way to the permittivity or the transmittance of the object. On the other hand, TDM necessitates to illuminate the sample under various incident angles in a sequential manner (so that real-time imaging is difficult) but it is possible to obtain quantitatively the map of permittivity (or transmittance) of the sample. Moreover, under the single scattering approximation and without any a priori information on the sample, the resolution of TDM should be better than that of wide-field microscopy.

\section{Techniques and perspectives of Tomographic Diffractive Microscopy}

In this section, we describe different experimental configurations for Tomographic Diffractive Microscopy. These techniques have often been presented under other names (microtomography, digital holographic tomography, phase tomography, synthetic aperture microscopy...). Basically, we found relevant for this review any optical imaging system devoted to the study of microscopic objects in which the sample is illuminated sequentially with various illuminations and the image is obtained via an inversion procedure from the phase and amplitude of the measured diffracted field. Note that, although the principles of TDM have been known for a long time [5], convincing experimental results with clear improvement of the resolution have been published only recently. 


\subsection{Experimental set-ups and applications}

Most TDM set-ups are implemented in standard reflection or transmission microscopes, such as the one schematically depicted in Fig. 4, with a coherent plane wave illumination. For a given illumination, the magnification of the microscope permits to measure on the CCD camera the diffracted field within a wide solid angle and with an appropriate sampling (one must satisfy the Shannon theorem). This approach is more convenient than a lensless "scatterometer" technique in which, for example, the diffracted field is measured by a detector placed on a rotating arm. In the following, we first describe several experimental configurations of TDM and discuss their power of resolution. Then, we present some research avenues for further developments.

\subsubsection{Phase measurements-Digital Holography Microscopy}

TDM set-ups differ from classical microscopes in that they measure both the phase and amplitude of the diffracted field, i. e. they record a hologram of the sample. When only one illumination is used, this approach is known as Digital Holographic Microscopy (DHM). DHM has stirred a lot of research in the last ten years because most applications developed in holography for macroscopic objects can be replicated at a microscopic scale with this novel technique. In this paper, we consider DHM as the simplest implementation of TDM and just recall its principles and limitations.

The main experimental difficulty of Digital Holography (and TDM) is to obtain a precise measurement of the phase of the diffracted field. Basically, this is done by introducing an interferometric set-up at the image [10] or Fourier [11] or even object [12] plane of the microscope. Currently, three methods have been used with great success. Phase stepping holographic microscopy consists in acquiring several holograms of the same specimen, with a controlled variation of the phase of the reference beam. Simple mathematics permits then to reconstruct the complex 
amplitude [13-15]. This technique has been used by several authors, both in holography and tomography. Its advantage is that the whole detector area contributes to the data acquisition. On the other hand, it is a slow technique as several successive holograms need to be recorded. An interesting alternative is provided by off-axis holography [16]. In this configuration, the reference beam is tilted with respect to the optical axis of the acquisition system. In that case, only one hologram is to be recorded, which is very favourable in terms of sensitivity to vibrations during acquisition. The resulting hologram fringes must be decoded [17], and a larger sensor has to be used. Care has to be taken for high numerical systems, in order to avoid mixing of the actual object frequencies with twin images appearing in this configuration. Heterodyne detection is another possibility $[18,19]$. This technique is extremely effective in the case of weakly diffracting specimens, when the diffracted field is small compared to the illumination wave, reaching the shot-noise detection limit.

These approaches are however indirect ones, as they reconstruct the complex field with the help of a well-characterized reference beam. Direct determination of the phase and amplitude is possible using front wave analysers like Shark-Hartman sensors, which may greatly simplify the acquisition setup by avoiding the need of a reference beam. Presently, these sensors are however still limited in terms of resolution (number of pixels), compared to CCD or CMOS cameras. One may also use numerical reconstructions of the phase and amplitude from one or several intensity-only measurements [20-23]. Finally, it is worth recalling that DHM (and TDM) are quantitative imaging tools. Hence they require a thorough calibration in order to accurately model the link between the sample and the measured data. The 
calibration procedure, which is usually performed with a well-known test object, can also be used to correct the possible aberrations of the optical system $[24,25]$.

The main interest of DHM is that it permits via Eq. (6) to retrieve threedimensional information on the sample from two-dimensional complex data that are obtained in a single shot. Generally, the sample is illuminated along the optical axis and the field is recorded in transmission within the solid angle of the microscope objective. The spatial frequencies of the object are known within a sphere cap, which is extended laterally but very narrow axially. An example of the object frequency support accessible using DHM is given in Fig. 5. As a result, Digital Holographic Microscopy, while having a rather good lateral resolution exhibits a limited sectioning capability along the optical axis [10,26,27].

Tomographic Diffractive Microscopy aims at increasing the accessible object frequency support (and consequently improving the resolution) by varying the illumination. Several approaches are possible, which we briefly describe now.

\subsubsection{Tomography with illumination rotation in transmission}

The most common configuration of TDM consists in illuminating the sample under various angles of incidence in a transmission microscope mode. Figure 6 shows how the synthetic aperture process induced by rotating the illumination $[11,28]$ enlarges the accessible object frequency domain. For the sake of simplicity, we consider here that the illumination and detection numerical apertures are identical. Other cases have been described in Ref [9].

Figure 6. Construction of the object frequency support (or Optical Transfer Function) that is accessible by rotating the illumination in a transmission holographic microscope. (a,b,c,d) longitudinal view, $(e, f, g, h)$ transverse view. $(a, e)$ Digital Holography Microscopy under normal incidence; $(b, f)$ oblique incidence $(c, g)$ DHM under oblique incidence; $(\mathrm{d}, \mathrm{h})$ object frequency support for height illuminations corresponding to the two maximum polar angles allowed by the numerical aperture of the condenser and four azimuth angles varying from 0 to $360^{\circ}$ every $45^{\circ}$ 
When a large number of incidences is used, in the $\left(k_{x}, k_{y}\right)$ plane, the detected frequencies fill the disk limited by the bold circle in Fig. 6(h). Note that the support of the object recorded frequencies obtained by varying the illumination angle, bold circle in Fig. 6(h), is twice as large as that given by digital holographic microscopy, for which only the normal incidence is used, see dotted circle in Fig. 6(h).

In the $\left(k_{x}, k_{z}\right)$ plane, the recorded object frequency support scanned by transmission TDM when a large number of incident angles is used has a more complex shape. It fills the butterfly shape depicted in Fig. 6(d). A detailed construction of this frequency support has been given in [29].

To assess the interest of transmission TDM with illumination rotation in terms of resolution, we compare in Fig. 7 its optical transfer function to that of Digital Holography Microscopy and wide-field transmission microscope. The lateral and axial extensions of the frequency supports for holographic microscopy and transmission TDM are given by [11].

$$
\Delta v_{x, y}^{\text {Holo }}=\frac{2 n \sin \theta}{\lambda} \Delta v_{z}^{\text {Holo }}=\frac{n(1-\cos \theta)}{\lambda} \text { and } \Delta v_{x, y}^{\text {Tomo }}=\frac{4 n \sin \theta}{\lambda} \Delta v_{z}^{\text {Tomo }}=\frac{2 n(1-\cos \theta)}{\lambda}
$$

We recall that the frequency support of transmission TDM is the same as that of a classical transmission microscope if the same condenser, objective and wavelength are used. However, in conventional transmission microscopy, the high frequencies are strongly attenuated (Figure 7(b) dotted line) while they are theoretically transmitted unaffected in TDM, (Figure 7(b) dashed line). The amelioration of the resolution, using transmission TDM with illumination rotation, compared to that of a conventional widefield microscope has been experimentally demonstrated in $[11,28,30]$.

Yet, this achievement is obtained at a certain expense. The main issue is the necessity of measuring, for numerous successive illuminations, the amplitude and phase of the diffracted field. While the classical transmission microscope with incoherent light may be considered as a parallel information processing system, the 
specimen being simultaneously illuminated with all the incidence angles allowed by the condenser, transmission TDM is a sequential information processing system. As a consequence, the technique may be slow, if mechanical movements of the specimen or of tilting mirrors are involved [10,11,27,28]. A simplified, higher-speed variant of this approach using a 1-D scanning only has been proposed to address this issue [31]. In that case, the recorded frequency support takes the characteristic shape of a "peanut" [32] and less object frequencies are recorded. This approach has permitted very fast acquisition, rendering the study of living specimens possible [31,33-35]. Another approach, which avoids rotating the illumination, consists in projecting a pattern (i. e. the sum of different plane waves) on the object and retrieving the information with a demodulating process in the image plane [36,37]. High-speed cameras (several thousands images per second) and beam control system that are now available should permit to record the large number of holograms mandatory to improve the resolution $[28,30]$ in a very short time, opening the way to high-speed, high resolution imaging of living specimens.

Last, it is worth noting that the resolution of transmission TDM with illumination rotation is not isotropic. Indeed, the recorded frequency support of transmission TDM presents a so-called "missing cone" along the optical axis, characteristic of transmission microscopes, which yields a relatively low resolution along this axis $[10,11,27,28,38,39]$. This important issue has been addressed and partially solved with other imaging configurations.

\subsubsection{Tomography with specimen rotation}

One solution to obtain a quasi-isotropic power of resolution consists in rotating the sample while keeping the illumination and detection static. Figure 8 describes the 
object frequency support in the case of transmission TDM with a complete specimen rotation about the $x$-axis and a plane wave illumination along the $z$-axis [40-42].

The advantage of this technique is that the holographic set-up is static and the power of resolution of the imager is quasi-isotropic. Most inversion algorithms used in this configuration are based on Radon transform [43-46], which is a simplified reconstruction procedure that neglects diffraction. This crude modelling of the experiment may have negative influence onto the specimen reconstruction $[47,48]$. Using more rigorous inversion schemes based on three-dimensional inverse Fourier transform improves the results $[47,48]$. This approach permits a quasi-isotropic resolution, with only a small, missing Fourier domain along the specimen rotation axis [42]. The main difficulty of this technique is to perform a precise rotation of the sample at the microscopic scale that is compatible with interferometric measurements. Manipulating the sample within a rotating microcapillary is not favoured by biologists, as handling microscopic specimens between a glass slide and a coverglass is easier. Techniques based on optical tweezers [49] or on electric fields [50] seem promising, but have not been used yet for interferometric measurements. As a consequence, one would often in practice prefer to keep the specimen static.

\subsubsection{Tomography with illumination rotation in reflection}

Improving the axial resolution can also be done by implementing a Tomographic Diffractive Microscopy set-up in a reflection microscope. Figure 9 describes the object frequency support that is accessible using reflection TDM with illumination rotation. The set of recorded frequencies in reflection TDM depicts a portion of a filled sphere, with the same lateral extension than that obtained in transmission TDM, but now comprising high frequencies along the optical axis, see Figure 9(c). From this construction, one therefore predicts that reflection TDM should be characterized by 
superior sectioning capabilities along the optical axis, which has been recently experimentally demonstrated [51]. Note that reflection configurations have often been used to study 2-D or quasi 2-D objects [7, 52-54] under paraxial approximation (which corresponds to low numerical aperture), see Eq. (7). In this case, the inversion procedure is greatly simplified since a two-dimensional inverse Fourier transform of the joined holograms yields the reflectance of the sample [7, 52-54]. This approximation amounts to replace the cap of spheres depicted in Fig. 9 by disks in the $\left(k_{\mathrm{x}}, k_{\mathrm{y}}\right)$ space that are placed at $k_{\mathrm{z}}=k_{0}$, a simplification which is also valid for transmission set-ups $[8,12,55,56]$.

\subsubsection{Tomography with illumination wavelength variation}

A radically different implementation of Tomographic Diffractive Microscopy consists in varying the illumination wavelength instead of varying the illumination angle.

Figure 10 gives the object frequency support obtained, in the case of fixed specimen, illumination and detection systems, for two wavelengths $\lambda_{1}$ and $\lambda_{2}=2 \lambda_{1}$, and for a transmission and a reflection set-up.

In the transmission configuration, Figure 10(a), both recorded caps of sphere have their summit at the frequency origin. Their curvature and extension change with the incident wavelength. This technique gives access to a rather limited frequency range. On the contrary, in the reflection configuration Figure 10(b), the accessible caps of sphere are placed at very different spatial locations in the Fourier space when the incident wavelength is changed. As a result the frequency support is significantly enlarged when the wavelength is varied from $\lambda_{1}$ to $\lambda_{2}$.

Figure 10(c) compares the frequency supports, that are obtained in transmission and reflection modes when the wavelength is continuously varied between $\lambda_{1}$ and $\lambda_{2}=2 \lambda_{1}$. It explains why wavelength scanning is performed 
preferentially in the reflection mode, either for 3-D tomography [57] or reflectometry [58-60]. In some cases, only a few wavelengths may be used in order to decouple index fluctuations from thickness variations $[61,62]$.

\subsection{Further developments}

In this paragraph, we focus on recent theoretical and, more rarely, experimental works, which should permit to enlarge the application domain of TDM and improve its power of resolution, possibly beyond the diffraction limit given by Eq. (10). Since Tomographic Diffractive Microscopy is a digital imaging technique, there are two main research avenues: improving the inversion algorithms or modifying the imaging configuration.

\subsubsection{Inversion algorithms}

Most inversion algorithms used in the framework of Tomographic Diffractive Microscopy assume a linear relation-ship between the scattered field and the parameter of interest (permittivity or reflectance). This assumption is particularly valid when the objects are weakly scattering (single scattering) and usually holds when biological samples are considered. Under the Born approximation $[5,10,11,26,27,63]$ or under the Rytov approximation $[34,64]$, it suffices to perform an inverse Fourier transform of the diffracted far-field to retrieve the permittivity map of the object. This widely used reconstruction procedure can be done in quasi real-time. Yet, it requires a tight regular sampling of the Ewald sphere and it is sensitive to noise and to the lack of data in certain frequency domains. Filtering is often used in order to dim artefacts such as Gibbs phenomenon or pixel scale effects [8,65]. A more sophisticated direct linear inversion method is based on the Singular Value Decomposition of the scattering operator. This approach permits to reconstruct objects from non-regularly sampled sparse data and to reduce the influence of noise 
on the reconstruction [66]. It may prove useful if one wants to diminish the number of illuminations in order to speed up the imaging process.

More recently, non-linear iterative inversion techniques have also been used to image highly contrasted samples $[67,68]$ such as those encountered in the nanotechnology domain. They provide much better images than that obtained with linear reconstruction algorithms when multiple scattering cannot be neglected [68] and they may even ameliorate the resolution beyond that expected with a single scattering analysis [69-71]. On the other hand, these iterative techniques are time consuming and cannot handle large set of data and unknowns. Up to now, they have been applied to objects whose size is comparable to the wavelength. Non-linear inversion techniques are traditionally expressed as optimization problems. The unknown map of permittivity within a given investigation domain $\Omega$ is parameterized and the value of the parameters is determined by minimizing a cost function. The latter is usually the $\mathrm{L}_{2}$ distance between the data and the field diffracted by an estimation of the permittivity map in $\Omega$. At each iteration, the diffracted field is rigorously computed from the previous estimate of the permittivity. The main bottleneck of this approach is that it requires repeated "exact" field computations, which may be excessively time-consuming. This has led to the emergence of modified-gradient [72,73] and contrast-source inversion methods [74,75], in which successive approximations of the permittivity and of the field inside $\Omega$ are obtained simultaneously [76]. The second important issue is convergence. Methods based on the optimization of a cost function inherently converge to a minimum, but that may be a local one. To avoid such minima, a quadratic approach may be used, as suggested in [77]. Another efficient method to circumvent the problem of local minima is to apply a regularization procedure, i. e. to introduce $a$ priori information on the object. 
The advantages of numerical imaging have still not been fully exploited.

Indeed, for the moment, most of the inversion techniques developed for TDM do not incorporate any a priori information on the object. It has been shown in the microwave domain that a spectacular amelioration of the images can be obtained by just specifying the lower and upper bounds of the sample permittivity [78]. In the optical domain, Sung and co-authors recently demonstrated that introducing the nonnegativity constraint on the permittivity in the reconstruction procedures yields a clear improvement of the image [34]. It permits to partially compensate for the missing cone in the object frequency support in transmission TDM with illumination rotation. A priori information on the object size is also very useful to improve the reconstruction [79]. Note that, when a priori information is introduced in the inversion algorithm, the resolution of the reconstruction can be much better than that imposed by the diffraction limit Eq. (10), even for weakly diffracting specimen [76, 79].

\subsubsection{Advanced imaging configurations}

In the best experimental configuration where the sample is illuminated and observed from every angle possible, one can theoretically obtain an isotropic resolution close to $0.35 \lambda$ if the single scattering approximation given by Eq. (10) is valid. Yet, most Tomographic Diffractive Microscopy set-ups are implemented in transmission or reflection microscopes where the sample is illuminated and/or observed from one side only. As a result the axial resolution is not as good as the transverse resolution. This major issue for three-dimensional imaging has stirred a lot of work in the microscopy community. Now, the digital nature and the sequential illumination process of Tomographic Diffractive Microscopy appear as definite assets to address this problem. Hence, to increase the angular coverage and improve the axial resolution, it 
is possible to rotate the sample, as seen in section 3.1.3 or to introduce a priori information in the inversion algorithms as seen in 3.2.1. Another recently put forward solution is to place the sample in the vicinity of a mirror in a reflection microscope. The mirror permits to retrieve simultaneously the top and bottom views of the object. The separation of these two components can easily be done using two incident polarizations or with a simple data processing [80]. As a result, one expects an isotropic resolution close to that given by Eq. (10).

Several studies have also been conducted to improve the transverse resolution of Tomographic Diffractive Microscopy. The simplest solution consists in diminishing the effective incident wavelength using immersion objectives or by depositing the sample onto a high index prism $[66,67]$. In the latter case, the inversion algorithms must be adapted to deal with evanescent waves, as one illuminates the sample under total internal reflection. The higher the refraction index $n$ of the immersion liquid or prism, the smaller the effective wavelength, $\lambda_{\mathrm{eff}}=\lambda / n$, and the better the resolution. With the present lossless materials existing in optics, the resolution can be improved by a factor of two at best. To overcome this limit, it has been proposed to deposit the sample on a periodically nanostructured substrate. The grating is optimized so as to transform the impinging propagative beam into an evanescent field with high spatial frequency. In this case, the effective wavelength of the field illuminating the sample depends solely on the grating period and it can be much smaller than $\lambda / 2$. Simulations of grating-assisted TDM have shown that one could expect from this approach a resolution about $\lambda / 10$ with a grating of period $\lambda / 5$ $[81,82]$. Note that this solution cannot be applied to analogical microscopes because the diffracted field is linked to the object in a complex way. 
Last, it is worth reporting another digital imager that permits to obtain a resolution well beyond the diffraction limit, the tomographic near-field microscope. In this approach, the sample is illuminated sequentially by a series of incident waves while the scattered field is measured in the near zone thanks to a scanning probe [8385]. An adapted inversion algorithm is then used to retrieve the three-dimensional map of permittivity of the sample with nanometric resolution.

\section{Conclusion}

Tomographic Diffractive Microscopy is a digital imaging technique that permits to study label-free samples. It consists in recording multiple holograms of the sample under different illuminations and using an inversion procedure to retrieve the sample parameters of interest. TDM exhibits a better power of resolution than that of classical microscopes and it yields quantitative information on the sample (such as the permittivity map) that is inaccessible with analogical microscopes. This approach belongs to the growing number of sequential digital imaging systems that combine sophisticated experimental set-ups with advanced numerical reconstructions (including, for example, a priori information on the sample). Digital imaging is mandatory in the microwave $[78,86], \mathrm{X}$-ray [87] or acoustic and seismic $[63,88]$ domains where lenses able to form analogically the image of a sample do not exist. It is also appearing in the active domain of fluorescence microscopy where recording multiple images of the same sample under various illuminations and using numerical reconstruction procedures permits to significantly improve the resolution [89-92]. In our opinion, the continuous progress in computing facilities, in high-speed detectors and wave front shaping (for varying the illumination) is a strong asset in favour of this 
new imaging approach, which, we believe, should undergo spectacular developments in a near future.

\section{Acknowledgements}

The authors would like to acknowledge Matthieu Debailleul and Bertrand Simon for enlightening discussions about optical diffractive tomography. 


\section{References}

[1] J.D. Jackson, Classical Electrodynamics, Wiley, New York (1999)

[2] M. Born and E. Wolf, Principles of Optics, Cambridge University Press, Cambridge (1999)

[3] X-ray and Neutron Reflectivitiy Principles and applications, Jean Daillant and Alain Gibaud (Eds), Lecture Notes in Physics, Springer, Berlin (1999)

[4] R. K. Mueller, M. Kaveh and G. Wade, Proc IEEE 1979, 67, 567- 587

[5] E. Wolf, Opt. Comm. 1969, 1, 153-156

[6] J. A. Ogilvy, Prog. Phys. 1987, 50, 1553-1603

[7] S. Alexandrov, T. Hillman, T. Gutzler, D. Sampson, Phys. Rev. Lett. 2006, 168102

[8] V. Mico, Z. Zalevski, P. Garcia-Martinez, J. Garcia, J. Opt. Soc. Am. A 2006, 3162-3170

[9] N. Streibl, J. Opt. Soc. Am. 1985, 2, 121-127

[10] M. Debailleul, B. Simon, V. Georges, O. Haeberlé, and V. Lauer, Meas. Sci. Technol. 2008, 19, 074009

[11] V. Lauer, J. Microsc. 2002, 205, 165-176

[12] V. Mico, Z. Zalevsky, and J. García, Opt. Comm. 2007, 276, 209-217

[13] W. Jueptner, U. Schnars, Digital Holography Digital Hologram Recording, Numerical Reconstruction, and Related Techniques, Springer, Berlin (2005)

[14] U. Schnars, J. Opt. Soc. Am. A 1994, 11, 2011-2015

[15] T. Zhang, and I. Yamaguchi,Opt. Lett. 1997, 22, 1268-1270

[16] E.N. Leith and J. Upatnieks. J. Opt. Soc. Am. 1962, 52, 1123-1128

[17] D. C. Ghiglia and M. D. Pritt Two-Dimenional Phase Unwrapping - Theory, Algorithms, and Software John Wiley \& Sons - New-York (1998)

[18] F. Le Clerc, L. Collot, and M. Gross, Opt. Lett. 2000, 25, 716-718

[19] F. Le Clerc, M. Gross, and L. Collot, Opt. Lett. 2001, 26, 1550-1552

[20] R.W. Gerchberg and W.O. Saxton, Optik 1972, 35, 237-246

[21] G. Liu and P.D. Scott, J. Opt. Soc. Am A, 1987, 4, 159-165

[22] A. Anand and B. Javidi, Opt. Lett. 2010, 35, 766-768

[23] A. Barty, K. A. Nugent, A. Roberts, D. Paganin, Opt. Comm. 2000, 175, 329-336

[24] T. Colomb, E. Cuche, F. Charrière, J. Kühn, N. Aspert, F. Montfort, P. Marquet, and C. Depeursinge, Appl. Opt. 2006, 45, 851-863

[25] G. Maire, J. Girard, F. Drsek; H. Giovannini, A. Talneau, K. Belkebir; P. C. Chaumet, and A. Sentenac "Experimental inversion of optical diffraction tomography data with a nonlinear algorithm in the multiple scattering regime" J. Mod. Opt. (accepted 2010) DOI: 10.1080/09500341003599739

[26] B. Simon, M. Debailleul, V. Georges, V. Lauer, and O. Haeberlé, Eur. Phys. J. App. Phys.2008, 44, 29-35

[27] M. Debailleul, V. Georges, B. Simon, R. Morin and O. Haeberlé, Opt. Lett. 2009, 34, 79-81

[28] T. Noda, S. Kawata and S. Minami, Appl. Opt. 1992, 31, 670-674

[29] O. Haeberlé, A. Sentenac and H. Giovaninni, An introduction to diffractive tomographic microscopy, Modern Research and Educational Topics in Microscopy, Vol. II Editors: Antonio Mendez Vilas, Jesus Diaz Alvarez Formatex Badajoz, Spain (2007)

[30] B. Simon, M. Debailleul, A. Beghin, Y. Tourneur, O. Haeberlé, "High resolution tomographic diffractive microscopy of biological samples" J. Biophotonics (accepted 2010) DOI 10.1002/jbio.200900094 
[31] W. Choi, C. Fang-Yen, K. Badizadegan, S. Oh, N. Lue, R. R. Dasari, and M. S. Feld, Nature Meth. 2007, 4, 717-719

[32] S. S. Kou and C. J. R. Sheppard, Opt. Lett. 2008, 33, 2362-2364

[33] Y. Park, M. Diez-Silva, G. Popescu, G. Lykotrafitis, W. Choi, M. S. Feld, and S. Suresh, PNAS 2008, 105, 13730-13735

[34] Y. Sung, W. Choi, C. Fang-Yen, K. Badizadegan, R. R. Dasari, and M. S. Feld, Opt. Exp. 2009, 17, 266-277

[35] N. Lue, W. Choi, G. Popescu, K. Badizadegan, R. R. Dasari, and M. S. Feld, Opt Exp. 2008, 16, 16240-16246

[36] G. Indebetouw, P. Klysubun, T. Kim, and T.-C. Poon, J. Opt. Soc. Am. A 2000, $17,380-390$

[37] G. Indebetouw, A. El Maghnouji, and R. Foster, J. Opt. Soc. Am. A 2005, 22, 892-898

[38] R. Fiolka, K. Wicker, R. Heintzmann, and A. Stemmer, Opt. Exp. 2009, 17, 12407-12417

[39] B. Simon, M. Debailleul, V. Georges, O. Haeberlé and V. Lauer, Photonics Europe 2008, EPE105, Strasbourg, April 7-11 (2008), Proceeding SPIE vol 6991, paper 699112 (2008)

[40] A. C. Sullivan and R. R. McLeod, Opt. Exp. 2007, 15, 14202-14212

[41] S. Vertu, I. Yamada, J.-J. Delaunay, O. Haeberlé, J. Flügge, European Conference on Biomedical Optics, Munich (Germany), June 14-18 (2009) Proc. of SPIE Vol. 7390, paper 73901D (2009)

[42] S. Vertu, J.-J. Delaunay, I. Yamada and O. Haeberlé, Centr. Eur. J. of Phys. 2009, 7, 22-31

[43] M. Fauver, E. J. Seibel, J. R. Rahn, M. G. Meyer, F. W. Patten, T. Neumann and A. C. Nelson, Opt. Exp. 2005, 13, 4210-4223

[44] F. Charrière, N. Pavillon, T. Colomb, C. Depeursinge, T. J. Heger, E. A. D. Mitchell, P. Marquet, and B. Rappaz, Opt. Exp. 2006, 14, 7005-7013

[45] F. Charrière; A. Marian, F. Montfort, J. Kuehn, T. Colomb, E. Cuche,P. Marquet, C. Depeursinge, Opt. Lett. 2006, 31 178-180

[46] S.Vertu, M. Ochiai, M. Shuzo, I. Yamada, J.-J. Delaunay, O. Haeberlé, Y. Okamoto, European Conference on Biomedical Optics, Munich (Germany), June 15-22 (2007), Proc. SPIE vol 6627, paper 66271A (2007)

[47] W. Gorski and W. Osten, Opt. Lett. 2007, 32, 1977-1979

[48] S. Vertu, I. Yamada, J.-J. Delaunay, and O. Haeberlé, Photonics West 2008, San Jose, January 19-24 (2008), Proc. SPIE Vol. 6861, paper 686103 (2008)

[49] M. K. Kreysing, T. Kießling, A. Fritsch, C. Dietrich, J. R. Guck, and J. A. Käs, Opt. Exp. 2008, 16, 16984-16992

[50] B. Le Saux, B. Chalmond, Y. Yu, A. Trouvé, O. Renaud and S.L. Shorte, J. Microsc. 2009, 233, 404-416

[51] M. Sarmis, B. Simon, M. Debailleul, B. Colicchio, V. Georges, J.-J. Delaunay and O. Haeberlé, "High resolution reflection tomographic diffractive microscopy" J. Mod. Opt. (accepted 2010) DOI: 10.1080/09500341003624743

[52] M. Paturzo, F. Merola1, S. Grilli, S. De Nicola, A. Finizio, and P. Ferraro Opt. Exp. 2008, 16, 17107-17118

[53] J. H. Massig, Opt. Lett.2002, 27, 2179-2181

[54] C. Liu, Z. Liu, F. Bo, Y. Wang, and J. Zhu, Appl. Phys. Lett.2002, 81, 3143

[55] L. Martínez-León and B. Javidi, Opt. Exp. 2008, 16, 161-169

[56] V. Mico, Z. Zalevsky, and J. García, Opt. Comm. 2008, 281, 4273-4281 
[57] J. Kühn, F. Montfort, T. Colomb, B. Rappaz, C. Moratal, N. Pavillon, P. Marquet, and C. Depeursinge, Opt. Lett. 2009, 34, 653-655

[58] F. Montfort, T. Colomb, F. Charrière, J. Kühn, P. Marquet, E. Cuche, S. Herminjard, and C. Depeursinge, Appl. Opt. 2006, 45, 8209-8217

[59] T. Colomb, S. Krivec, H. Hutter, A. A. Akatay, N. Pavillon, J. Kühn, C. Depeursinge, and Y. Emery, Opt. Exp. 2010, 18, 3719-3731

[60] P. Ferraro, L. Miccio, S. Grilli, M. Paturzo, S. De Nicola, A. Finizio, R. Osellame, and P. Laporta, Opt. Exp. 2007, 15, 14591-14600

[61] M.-K. Kim, Opt. Exp. 2000, 7, 305-310

[62] B. Rappaz, F. Charrière, C. Depeursinge, P. J. Magistretti, and P. Marquet, Opt. Lett. 2008, 33, 744-746

[63] A.J. Devaney, Ultrasonic Imaging, 1982, 4, 336-350

[64] B. Chen and J. Stammes, Appl. Opt. 1998, 37, 2996-3006

[65] T. R. Hillman, T. Gutzler, S. A. Alexandrov, and D. D. Sampson, Opt. Exp.2009, $17,7873-7892$

[66] P. Scott Carney and J. C. Schotland, J. Opt. Soc. Am. A 2003, 20, 542-547

[67] K. Belkebir, P. Chaumet and A. Sentenac, J. Opt. Soc. Am. A 2005, 22, 18891897

[68] G. Maire, F. Drsek, J. Girard, H. Giovannini, A. Talneau, D. Konan, K. Belkebir, P. C. Chaumet and A. Sentenac, Phys. Rev. Lett. 2009, 102, 213905

[69] K. Belkebir, P. C. Chaumet and A. Sentenac, J. Opt. Soc. Am. A 2006, 23, 586596

[70] F. Simonetti, Phys. Rev. E 73, 036619 (2006)

[71] A. Sentenac, C.-A. Guérin, P. C. Chaumet, F. Drsek, H. Giovannini, N. Bertaux, and M. Holschneider, Opt. Exp. 2007, 15, 1340-1347

[72] R. E. Kleinman, and P. M. van den Berg, J. Comput. Appl. Math. 1992, 42, 17 35

[73] K. Belkebir, R. E. Kleinman, and C. Pichot, IEEE Trans. Microwave Theory and Techniques 1997, 45, 469-476

[74] P. M. van den Berg, and R. E. Kleinman, Inverse Probl. 1997, 13, 1607-1620

[75] A. Abubakar, and P. M. van den Berg, Inverse Probl. 2002, 18, 495-510

[76] P. M. Van Den Berg and R. E. Kleinman, Gradient methods in inverse acoustic and electromagnetic scattering, in Large-Scale Optimization with Applications, Part I: Optimization in Inverse Problems and Design, L. T. Biegler, et. al. eds. IMA Volumes in Mathematics and its Applications. Vol. 92, Springer-Verlag, New York, 1997, 173-194.

[77] R. Pierri, G. Leone, IEEE Trans. Geosci. Remote Sensing 1999, 37, 374- 382

[78] P. C. Chaumet, K. Belkebir and A. Sentenac, J. Appl. Phys. 2009, 109, 034901

[79] P. C. Chaumet, K. Belkebir and A. Sentenac, Phys. Rev. B 2004, 69, 245405

[80] E. Mudry, P. C. Chaumet, K. Belkebir, G. Maire and A. Sentenac, "Mirrorassisted Tomographic Diffractive Microscopy with isotropic resolution", Opt. Lett. (submitted).

[81] A. Sentenac, P. Chaumet, and K. Belkebir, Phys. Rev. Lett. 2006, 24, 243901

[82] P. Chaumet, K. Belkebir and A. Sentenac, Phys. Rev. A 2007,76, 013814

[83] P. Scott Carney and J. C. Schotland, Appl. Phys. Lett. 2000, 77, 2798-2800

[84] P. Scott Carney, V. A. Markel, and J. C. Schotland, Phys. Rev. Lett. 2000, 86, 5874-5877

[85] P. Scott Carney, R. A. Frazin, S. I. Bozhevolnyi, V. S. Volkov, A. Boltasseva, and J. C. Schotland, Phys. Rev. Lett. 2004, 92, 163903 
[86] M. Slaney, A. C. Kak, and L. E. Larsen, IEEE Trans. Micro. Theory and Techniques 1984, MTT-32, 860-874

[87] I. McNulty, J. Kirz, C. Jacobsen, E. H. Anderson, M. R. Howells, and D. P. Kern, Science 1992, 256, 1009-1012

[88] R.-S. Wu and M. Nafi Toksöz, Geophysics 1987, 52, 11-25

[89] R. Heintzmann, T. M. Jovin, and C. Cremer, J. Opt. Soc. Am. A 2002, 19, 15991609

[90] M. G. L. Gustafsson, PNAS, 2005, 102, 13081-13086

[91] B. Huang, W. Wang, M. Bates, and X. Zhuang, Science 2008, 319, 810-813

[92] E. Betzig, G. H. Patterson, R. Sougrat, O. W. Lindwasser, S. Olenych, J. S. Bonifacino, M. W. Davidson, J. Lippincott-Schwartz and H. F. Hess, Science 2006, 313, 1642-1645 


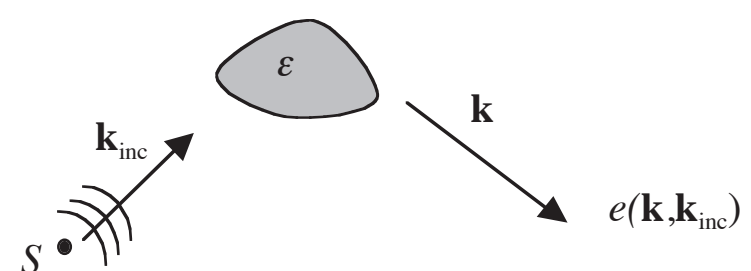

Figure 1. A plane wave with wavevector $\mathbf{k}_{\text {inc }}$ illuminates an object characterized by its relative permittivity $\varepsilon$. The detected diffracted field in the direction $\mathbf{k}$ is given by $\mathbf{e}\left(\mathbf{k}, \mathbf{k}_{\text {inc }}\right)$. 

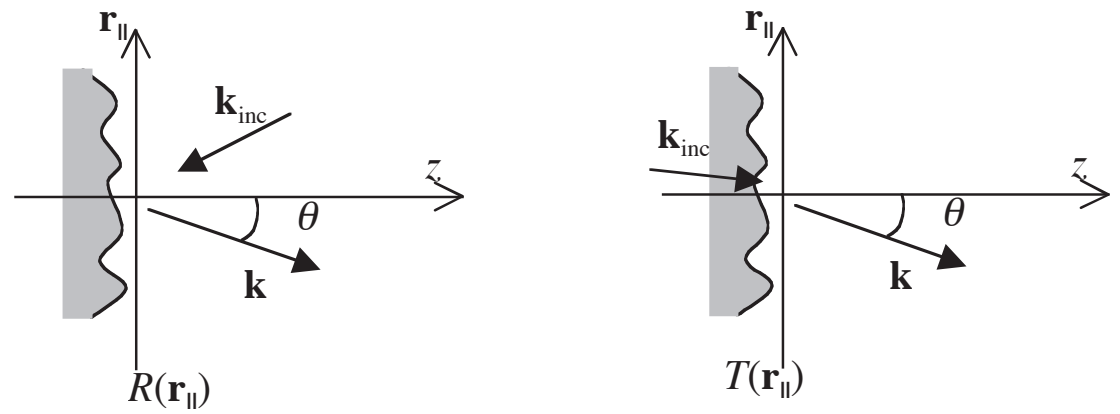

Figure 2. Different imaging configurations. Left :Reflection configuration, the object is defined by its reflectance $\mathrm{R}\left(\mathbf{r}_{\|}\right)$. Right transmission configuration, the object is defined by its transmittance $\mathrm{T}\left(\mathbf{r}_{\|}\right)$. 


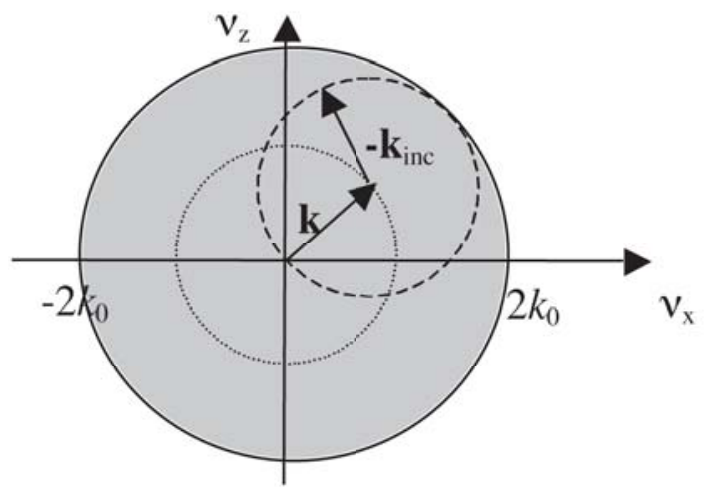

Figure 3. The Fourier domain of the sample permittivity that is accessible at the best, $i$. e.when the sample is illuminated and observed from every angle possible. . 


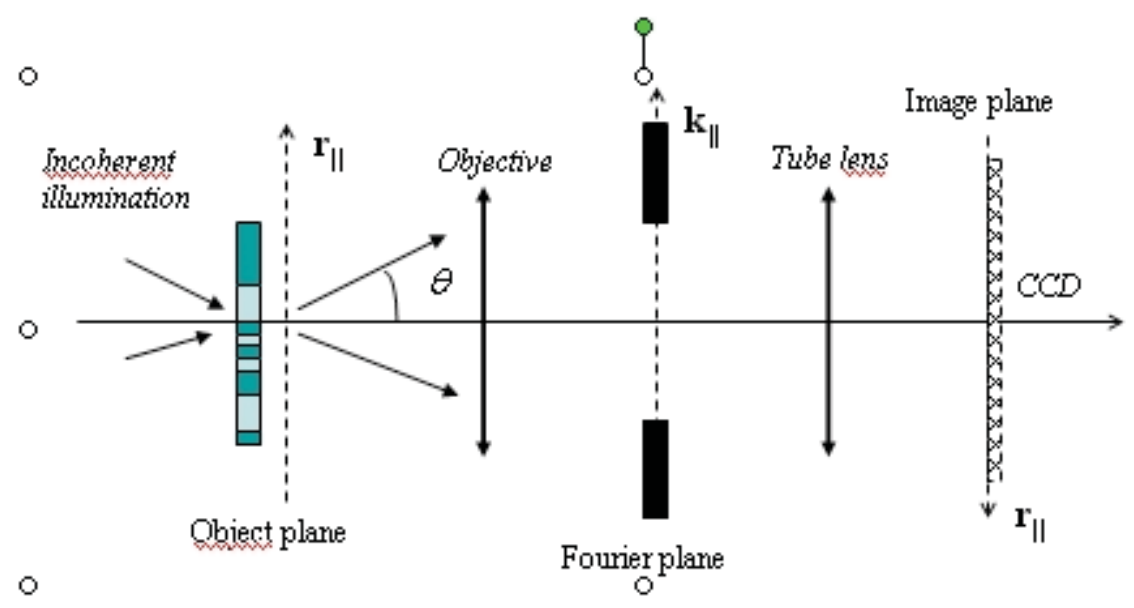

Figure 4. Principle of detection of the diffracted wave in a classical transmission microscope. 

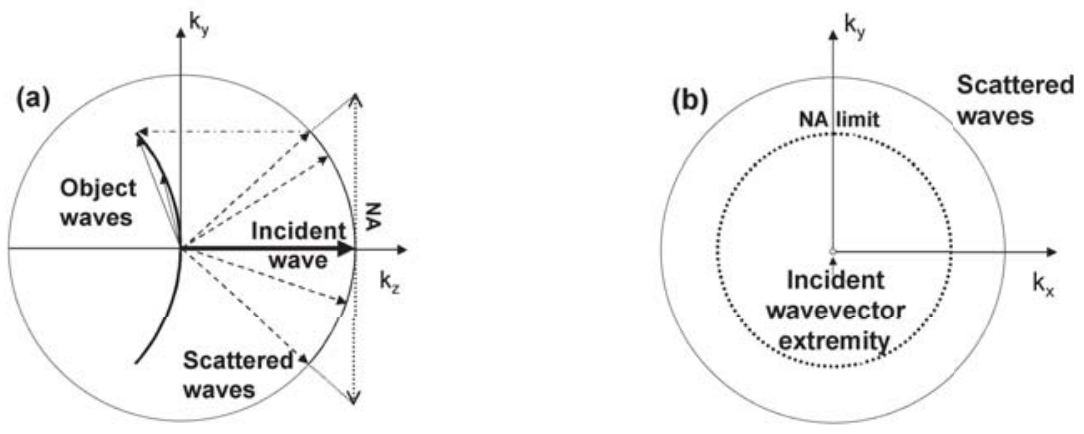

Figure 5. Construction of the set of object detected frequencies in the Fourier space in the case of digital holographic microscopy. (a): longitudinal view, and (b): transverse view. 

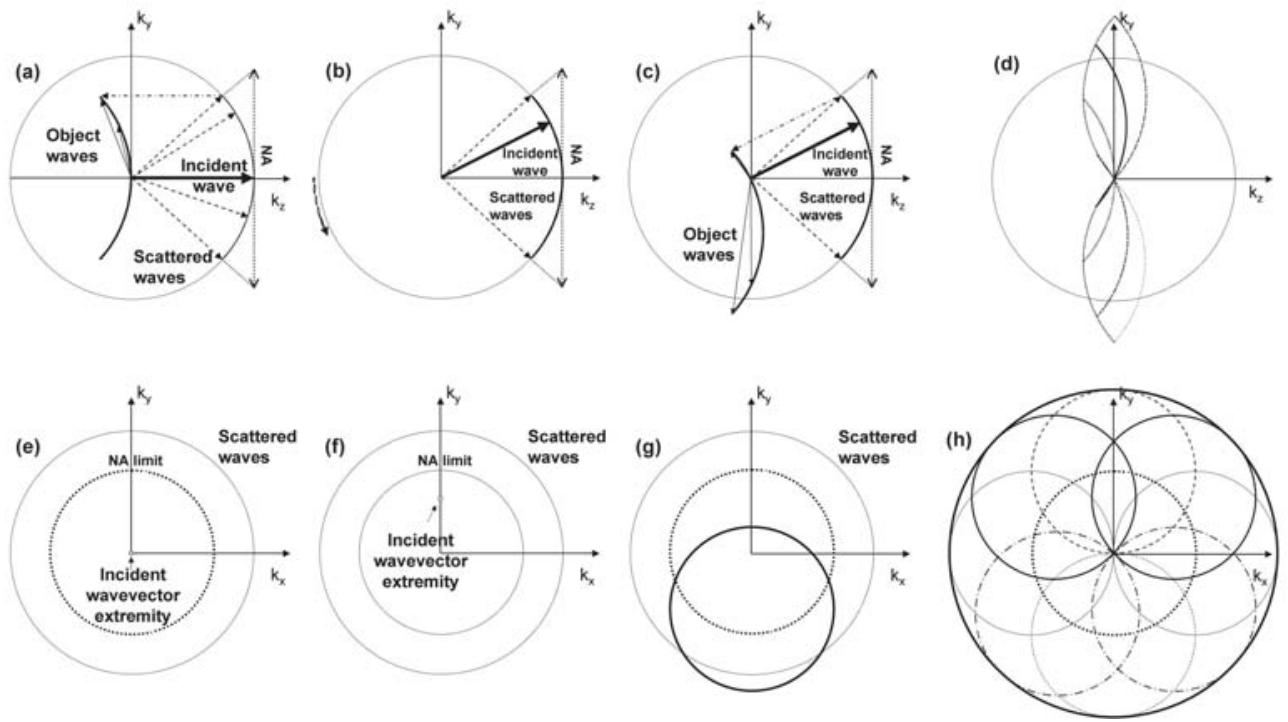

Figure 6. Construction of the object frequency support (or Optical Transfer Function) that is accessible by rotating the illumination in a transmission holographic microscope. (a,b,c,d) longitudinal view, $(\mathrm{e}, \mathrm{f}, \mathrm{g}, \mathrm{h})$ transverse view. $(\mathrm{a}, \mathrm{e})$ Digital Holography Microscopy under normal incidence; $(b, f)$ oblique incidence $(c, g)$ DHM under oblique incidence; $(\mathrm{d}, \mathrm{h})$ object frequency support for height illuminations corresponding to the two maximum polar angles allowed by the numerical aperture of the condenser and four azimuth angles varying from 0 to $360^{\circ}$ every $45^{\circ}$ 


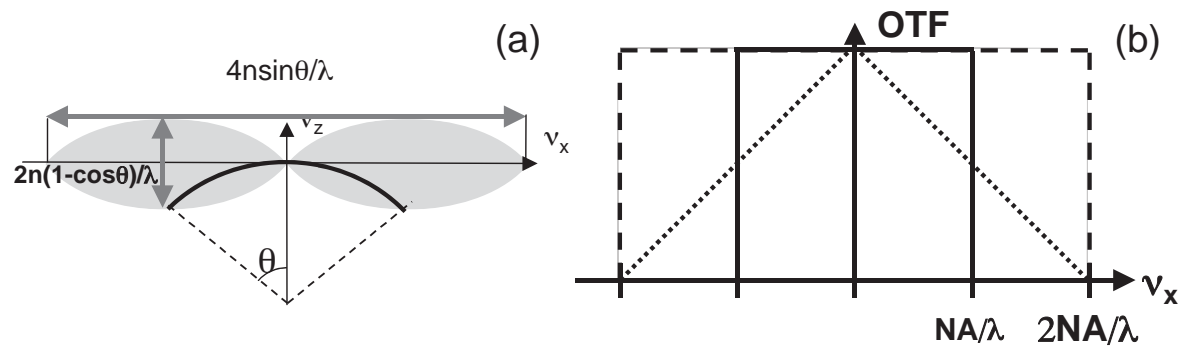

Figure 7. (a) Comparison of frequency supports for holography (solid line) and TDM in transmission with illumination rotation (in grey), longitudinal view. (b): Comparison of Optical Transfer Functions for incoherent transmission microscopy (dotted line), holographic microscopy (solid line), and transmission TDM (dashed line). Note that in (b), for the sake of simplicity, the geometry is assumed to be invariant along the $y$ axis. 

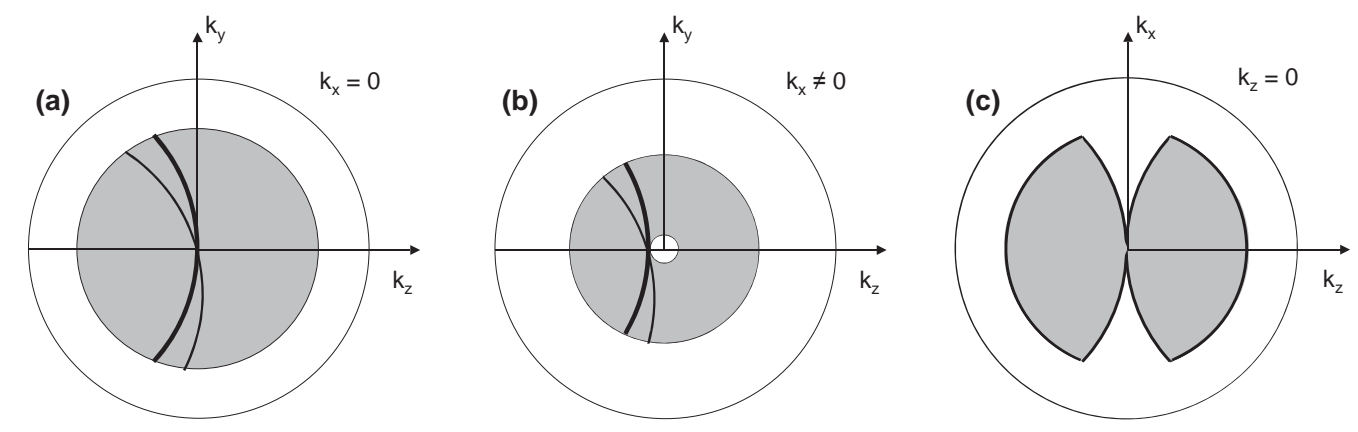

Figure 8. Tomographic Diffractive Microscopy in transmission: construction of the set of detected waves for specimen rotation. The optical transfer function is quasi-isotropic. (a) thick solid line: frequency support in the $\mathrm{k}_{\mathrm{x}}=0$ plane obtained for the initial position of the sample, thin solid line : frequency support obtained after one rotation of the sample about the $\mathrm{x}$-axis. Gray disk: frequency support obtained when the sample is rotated continuously over $360^{\circ}$.(b) idem (a) but in the $k_{\mathrm{x}} \neq 0$ plane (c) idem (a) in the $k_{\mathrm{y}}=0$ plane 

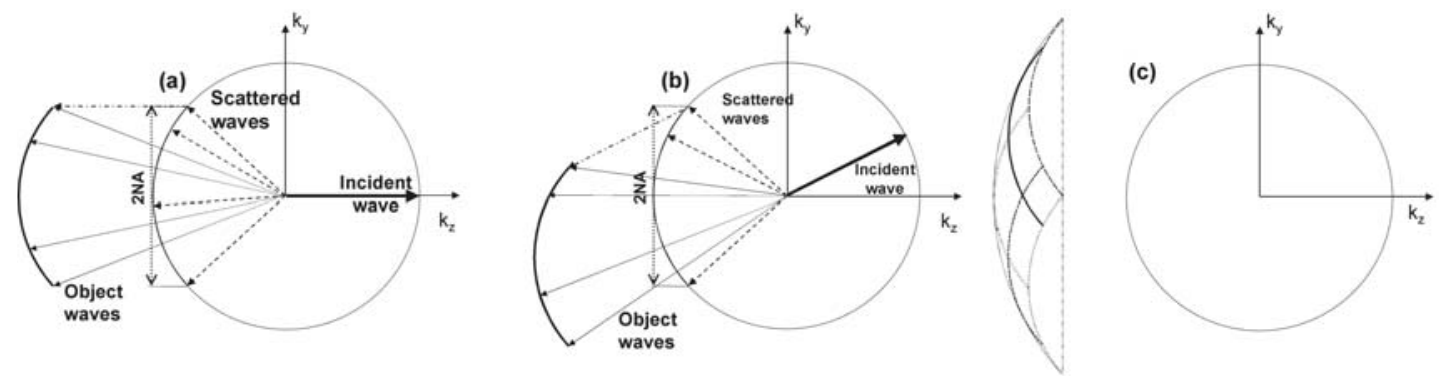

Figure 9. Reflection TDM with illumination rotation: construction of the object frequency support, longitudinal view to be compared to Fig. 6(a,c,d). 

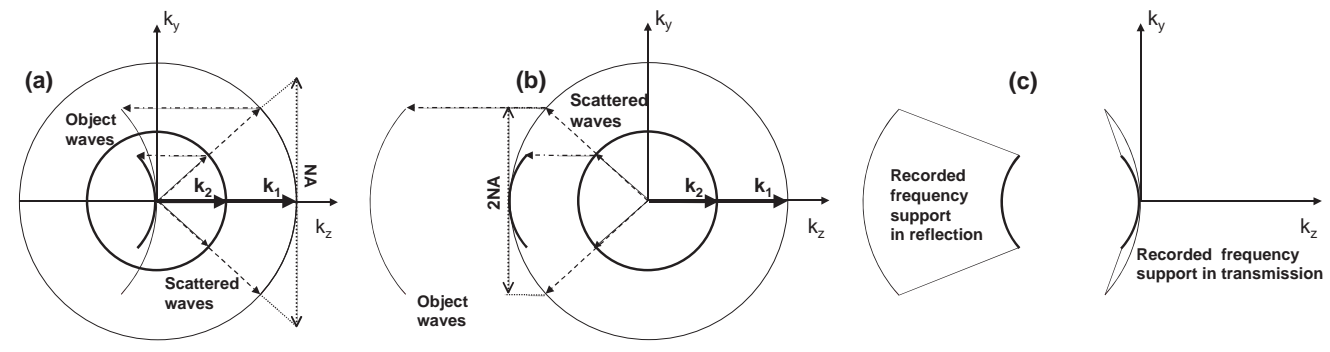

Figure 10. TDM with illumination wavelength variation: construction of the object frequency support in transmission and reflection modes. 